\title{
MuLV-related endogenous retroviral elements and Flt3 participate in aberrant end-joining events that promote B-cell leukemogenesis
}

\author{
Radia M. Johnson, ${ }^{1,2,6}$ Eniko Papp, ${ }^{1,3,6}$ Ildiko Grandal, ${ }^{1}$ Paul E. Kowalski, ${ }^{1}$ Lauryl Nutter, ${ }^{1}$ \\ Raymond C.C. Wong, ${ }^{4}$ Ann M. Joseph-George, ${ }^{4,5}$ Jayne S. Danska, ${ }^{2,3,5,6}$ and Cynthia J. Guidos ${ }^{1,2,6,7}$ \\ ${ }^{1}$ Program in Developmental and Stem Cell Biology, Hospital for Sick Children Research Institute, Toronto, Ontario M5G 0A4, \\ Canada; ${ }^{2}$ Department of Immunology, ${ }^{3}$ Department of Medical Biophysics, University of Toronto, Toronto, Ontario M5S $1 \mathrm{~A} 8$, \\ Canada; ${ }^{4}$ The Centre for Applied Genomics, Hospital for Sick Children Research Institute, Toronto, Ontario M5G 0A4, Canada; \\ ${ }^{5}$ Program in Genetics and Genome Biology, Hospital for Sick Children Research Institute, Toronto, Ontario M5G 0A4, Canada
}

\begin{abstract}
During $V(D) J$ recombination of immunoglobulin genes, p53 and nonhomologous end-joining (NHEJ) suppress aberrant rejoining of DNA double-strand breaks induced by recombinase-activating genes (Rags)-1/2, thus maintaining genomic stability and limiting malignant transformation during B-cell development. However, Rag deficiency does not prevent B-cell leukemogenesis in p53/NHEJ mutant mice, revealing that p53 and NHEJ also suppress Rag-independent mechanisms of B-cell leukemogenesis. Using several cytogenomic approaches, we identified a novel class of activating mutations in Fms-like tyrosine kinase 3 (FIt3), a receptor tyrosine kinase important for normal hematopoiesis in Rag/p53/NHEJ triple-mutant (TM) B-cell leukemias. These mutant Flt3 alleles were created by complex genomic rearrangements with Moloney leukemia virus (MuLV)-related endogenous retroviral (ERV) elements, generating ERV-Flt3 fusion genes encoding an N-terminally truncated mutant form of Flt3 (trFlt3) that was transcribed from ERV long terminal repeats. trFlt3 protein lacked most of the Flt3 extracellular domain and induced ligand-independent STAT5 phosphorylation and proliferation of hematopoietic progenitor cells. Furthermore, expression of trFlt3 in p53/NHEJ mutant hematopoietic progenitor cells promoted development of clinically aggressive B-cell leukemia. Thus, repetitive MuLV-related ERV sequences can participate in aberrant end-joining events that promote development of aggressive B-cell leukemia.
\end{abstract}

[Keywords: nonhomologous end-joining; p53; B-cell development; endogenous retrovirus; Fms-like tyrosine kinase 3; lymphoblastic leukemia]

Supplemental material is available for this article.

Received March 1, 2014; revised version accepted April 25, 2014.

Development of precursor B-cell acute lymphoblastic leukemia (B-ALL) is driven by mutations in transcription factors and cytokine signaling cascades that regulate key steps in B-cell development. For example, Fms-like tyrosine kinase 3 (Flt3), interleukin-7 receptor, and Pax5 promote B-cell progenitor survival, proliferation, specification, and commitment and are frequently mutated in B-ALL (Inaba et al. 2013). PAX5 induces B-cell commitment and Cd19 expression while repressing Flt3 transcription (Holmes et al. 2006), rendering CD19 ${ }^{+}$B-cell progenitors insensitive to Flt3 ligand (FL), a ubiquitously expressed growth-promoting cytokine. Subsequent proliferation

\footnotetext{
${ }^{6}$ These authors contributed equally to this work.

${ }^{7}$ Corresponding author

E-mail cynthia.guidos@sickkids.ca

Article is online at http://www.genesdev.org/cgi/doi/10.1101/gad.240820.114.
}

and differentiation requires somatic assembly of immunoglobulin heavy chain (Igh) genes initiated by the recombinase-activating gene (Rag)-encoded endonuclease. Rag induces DNA double-strand breaks (DSBs) adjacent to $V, \mathrm{D}$, and $J$ gene segments in the Igh locus that are repaired by the DNA-dependent protein kinase (Prkdc) and other ubiquitously expressed nonhomologous endjoining (NHEJ) components. However, Rag-induced DSBs can be aberrantly rejoined, resulting in chromosomal translocations and focal gene deletions that play a major role in B-ALL pathogenesis (Mullighan et al. 2008;

\footnotetext{
(C) 2014 Johnson et al. This article is distributed exclusively by Cold Spring Harbor Laboratory Press for the first six months after the full-issue publication date (see http://genesdev.cshlp.org/site/misc/terms.xhtml). After six months, it is available under a Creative Commons License (AttributionNonCommercial 4.0 International), as described at http://creativecommons. org/licenses/by-nc/4.0/.
} 
Papaemmanuil et al. 2014). Thus, Rag-induced DSBs represent a major threat to genomic stability during B-cell development.

p53 and NHEJ play important roles in suppressing oncogenic rearrangements of Rag-induced DSBs in B-cell progenitors. In NHEJ-deficient mice, Rag-induced DSBs persist abnormally and activate p53-dependent DNA damage responses that promote apoptotic elimination of lymphocyte progenitors undergoing $\mathrm{V}(\mathrm{D}) \mathrm{J}$ recombination (Guidos et al. 1996). In p53/NHEJ double-mutant (DM) mice, aberrant repair of Rag-induced DSBs generates Igh$c M y c$ rearrangements that promote malignant transformation of B-cell progenitors (Difilippantonio et al. 2002; Zhu et al. 2002; Gladdy et al. 2003). The telomeric location of $I g h$ (on chromosome 12) coupled with a general defect in telomere maintenance in NHEJ-deficient mice (d'Adda di Fagagna et al. 2004) causes Rag-induced DSBs to undergo end-to-end fusions with other chromosomes and participate in bridge-breakage-fusion cycles that generate complex chromosomal rearrangements (Difilippantonio et al. 2002; Zhu et al. 2002; Gladdy et al. 2003).

Surprisingly, however, genomically unstable B-ALLs develop with similar incidence and latency in DM versus Rag- $2^{-/-}$p53 ${ }^{-/-}$Prkdc scid/scid triple-mutant (TM) mice (Gladdy et al. 2003). Interestingly, TM but not DM B-ALLs showed frequent $(\sim 75 \%)$ dissemination to the central nervous system (CNS) (Gladdy et al. 2003), causing CNS pathologies similar to those seen in highrisk human B-ALL (Pui 2006). Thus, Rag-independent oncogenic drivers cause development of clinically aggressive B-ALLs in TM mice. Although p53 and NHEJ regulate DNA damage responses and DNA repair in all tissues, TM mice do not develop nonlymphoid malignancies. These findings suggest that B-cell precursors are uniquely susceptible to Rag-independent aberrant endjoining events that promote development of aggressive CNS-invasive precursor B-ALLs, but these have not been characterized.

The Rag-encoded endonuclease is thought to have evolved from a transposon that became "endogenized" in the mouse genome (Thompson 1995). It is now appreciated that $4 \%-10 \%$ of vertebrate genomes contain endogenous retrovirus (ERV) sequences, remnants of ancient germline retroviral infections (Stocking and Kozak 2008; Stoye 2012). Class I ERVs, present in 50-100 copies per genome, are closely related to $\gamma$-retroviruses such as exogenous Moloney leukemia virus (MuLV) with ecotropic, xenotropic, or polytropic host range (Stocking and Kozak 2008; Stoye 2012). Because ERVs are present in high copy number and include repeated sequences, they undergo frequent recombination-based rearrangements that have greatly contributed to remodeling of vertebrate genomes during evolution (Feschotte and Gilbert 2012). Infection of hematopoietic progenitors with exogenous MuLV promotes lymphoid leukemogenesis by deregulating expression of adjacent cellular genes (Uren et al. 2005). This mechanism depends on transcriptional promoters and enhancers located in MuLV-encoded long terminal repeats (LTRs) (Feschotte and Gilbert 2012). Although most MuLV-related ERVs have acquired mutations that preclude production of infectious virus, recombination between these repetitive elements can "reactivate" production of infectious ERVs that promote lymphoid leukemogenesis (Stoye et al. 1991; Young et al. 2012; $\mathrm{Yu}$ et al. 2012). However, the ability of noninfectious MuLV-like ERVs to participate in oncogenic chromosomal rearrangements is unknown.

In this study, we used cytogenomic approaches to identify Rag-independent mechanisms that promote leukemic transformation of B-cell precursors in TM mice. We identified a novel class of Flt3-activating mutations that were created by complex multistep chromosomal rearrangements with ERV elements. The Flt3 promoter and N-terminal exons encoding the ligand-binding domain were deleted and replaced with LTRs from several different MuLV-related ERVs. The resulting ERV-Flt3 fusion genes encoded constitutively active trFlt3 (an N-terminally truncated mutant form of Flt3) proteins with ligand-independent signaling properties similar to Flt3 internal tandem duplication (ITD) mutations that promote FL-independent FLT3 signaling in human B-ALL and acute myeloid leukemia (AML) (Stirewalt and Radich 2003). Importantly, ERV-Flt3 fusion genes were never detected in DM B-ALLs or in TM B-ALLs that lacked CNS dissemination. Furthermore, ectopic trFlt3 expression promoted rapid generation of CNS-disseminating B-ALLs from DM hematopoietic progenitors, demonstrating that aberrant Flt3 activation underlies the unique ability of B-ALLs arising in TM mice to invade the CNS. Collectively, these data demonstrate that repetitive MuLV-related ERV sequences can participate in aberrant end-joining events that promote development of aggressive B-cell leukemia.

\section{Results}

Recurrent alterations of chromosomes 2 and 5 in TM $B-A L L$

TM mice develop genomically unstable B-ALLs but lack Rag-induced Igh-cMyc translocations or other recurrent cytogenetic alterations detectable by spectral karyotype (SKY) analyses (Gladdy et al. 2003). Since SKY cannot detect alterations involving small chromosomal regions, we used array comparative genomic hybridization (aCGH) to search for recurrent copy number variations (CNVs) that might help identify oncogenic drivers that promote transformation of B-cell precursors into CNS-invasive B-ALLs. In a cohort of 10 TM B-ALLs isolated from TM mice showing clinical signs of CNS leukemia, we observed recurrent CNVs involving chromosomes 2 and/or 5, with a commonly deleted $15.6-\mathrm{Mb}$ telomeric region on the chromosome 2 region extending from $2 \mathrm{H} 3$ to $2 \mathrm{H} 4$ and a commonly amplified $11.5-\mathrm{Mb}$ telomeric region on chromosome 5 that included G2 to 5G3 (Fig. 1A). Interestingly, aCGH analysis of TM 5199 showed focal amplification of the chromosome 5 telomeric region containing Flt3 on the negative strand. Using a higher-resolution oligonucleotide array, we found that sequences 3 ' (centromeric) of Flt3 intron 10 were amplified, whereas sequences $5^{\prime}$ (telomeric) 

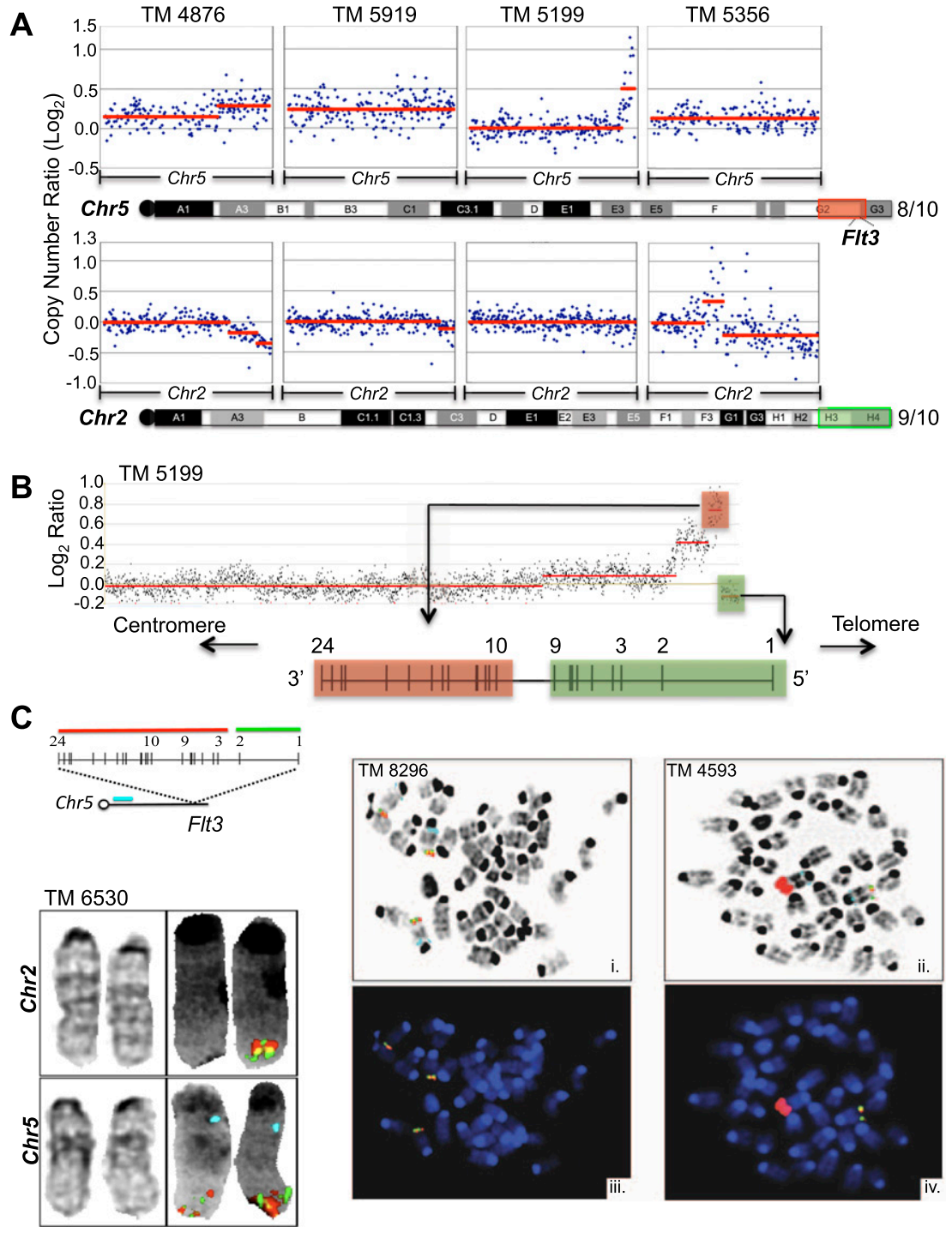

Figure 1. Cytogenomic analyses reveal recurrent chromosome 2 and 5 alterations in TM B-ALL. $(A, t o p)$ CNV results for TM BALLs using murine 6.5K RPCI-23/-24 BAC arrays. $\log _{2}$ ratio plots of leukemia/reference DNA signal (Cy3/Cy5) intensities (Y-axis) for each BAC probe (individual dots along the $X$ axis) from centromere to telomere (left to right) are shown for chromosomes 5 (top) and 2 (bottom) for four representative TM BALLs. Sliding window averages (red lines) were calculated by circular binary segmentation (CBS). (Bottom) Chromosome ideograms depict the minimal commonly amplified (red) or deleted (green) regions on chromosomes 5 and 2, based on analysis of 10 samples. (B) High-resolution CNV analysis of TM 5199 bone marrow (BM) using Nimblegen murine $385 \mathrm{~K}$ whole-genome array. $\log _{2}$ ratio of DNA signal intensities (Y-axis) is shown for chromosome 5 probes ( $X$-axis) from centromere to telomere. Sliding window averages (red line) were calculated using SegMNT (Roche NimbleGen). (C) Three types of chromosome 5 abnormalities identified by mFISH. (Top left) The chromosome 5 schematic shows the relative positions of "break-apart" probes spanning Flt3 exons 3-24 (red) and Flt3 exons 1-2 (green) as well as two centromeric chromosome 5 probes (blue). (Bottom left) mFISH overlay on G-banding of the dominant clone in TM 6530 shows nonreciprocal chromosome 5 translocation $(5 \mathrm{qG} 1 \sim 3$, including Flt3) to chromosome $2(2 \mathrm{G} \sim \mathrm{H})$. (Middle right) Representative inverted DAPI-banding (panels i,ii) and mFISH images (panels iii,iv) of dominant clones in TM 8296 and TM 4593. of Flt3 intron 9, which encode most of Flt3 extracellular region, were deleted (Fig. 1B).

To characterize chromosome 5 abnormalities in single cells and identify breakpoints within Flt3, we performed metaphase fluorescent in situ hybridization (mFISH) on six additional TM B-ALL samples using dual-color "break-apart" Flt3 probes together with additional probes specific for the centromeric region of chromosome 5 . We observed three cytogenetically distinct types of chromosome 5 abnormalities. TM 8296 is representative of four out of six samples exhibiting a dominant clone with simple trisomy for chromosome $5([+5])$ (Fig. 1C; Table 1). In contrast, TM 6530 showed a partial $[+5]$, with the telomeric region that includes Flt3 translocated to the telomeric end of chromosome 2, generating a derivative chromosome 2 [der(2)t(2;5)] (Fig. 1C). TM 9970 displayed both types of abnormalities, containing a dominant $[+5]$ clone and a subclonal $\operatorname{der}(2) \mathrm{t}(2 ; 5)$ (Table 1$)$. The $\operatorname{der}(2) \mathrm{t}(2 ; 5)$ subclone likely emerged from an initial $[+5]$ clone via a Robertsonian $\mathrm{t}(5 ; 14)$ (Supplemental Fig. S1A). Finally, the dominant TM 4593 clone exhibited a third type of partial [+5] involving extensive telomeric amplification of a region that included Flt3 exons 3-24 coupled with loss of exons 1-2 (Fig. 1C). However, TM 4593 also had other

Table 1. Frequency of chromosome 5 abnormalities

\begin{tabular}{lcccc}
\hline TM sample & Trisomy & $\operatorname{Der}(2) \mathrm{t}(2 ; 5)$ & Telomeric amp & Other \\
\hline 6129 & $\mathbf{8} / \mathbf{1 1}$ & & & \\
8296 & $\mathbf{1 0} / \mathbf{1 0}$ & & & \\
9962 & $\mathbf{1 2 / 1 9}$ & & & $1 / 18^{\mathbf{b}}$ \\
9970 & $\mathbf{1 5 / \mathbf { 1 8 } ^ { \mathbf { a } }}$ & $2 / 18$ & $\mathbf{1 1} / \mathbf{1 1}$ & \\
4593 & & $\mathbf{1 2} / \mathbf{1 2}$ & & \\
6530 & & &
\end{tabular}

Spleen or BM cells from leukemic TM mice were analyzed by metaphase three-color FISH using probes described for Figure 1C. The major clone for each sample is indicated in bold.

${ }^{\text {a }}$ Robertsonian $(5 ; 14)(\mathrm{A} 1 ; \mathrm{A} 1)$ in four of 15 spreads with +5 (trisomy).

${ }^{\mathrm{b}} \operatorname{Der}(\mathrm{X}) \mathrm{t}(\mathrm{X} ; 5)$ found in one of 18 spreads. 
Johnson et al.

subdominant clones exhibiting full [+5] or der(2)t(2;5) (Supplemental Fig. S1B). The occurrence of $[+5]$ together with $\operatorname{der}(2) \mathrm{t}(2 ; 5)$ and telomeric chromosome 5 amplification in distinct subclones of the same sample suggests ongoing clonal evolution, most likely involving bridge-breakagefusion events. Collectively, this clonal analysis demonstrated highly recurrent cytogenetic abnormalities of chromosomes 2 and 5 in CNS-invasive TM B-ALL samples.

\section{Leukemic B cells from TM mice with CNS signs overexpress truncated Flt3}

We next evaluated expression of Flt3 in TM B-ALL samples exhibiting these unusual N-terminal Flt3 deletions. Since $\sim 25 \%$ of TM mice develop B-ALLs that do not disseminate to the CNS (Gladdy et al. 2003), we compared TM B-ALL samples from mice with and without signs of CNS leukemia. CNS-invasive TM B-ALLs had 10-fold to 1000-fold higher levels of Flt3 mRNA than TM B-ALLs arising in mice without CNS signs or in DM mice (Fig. 2A). Most TM B-ALL samples expressing high levels of Flt3 mRNA exhibited low levels of cell surface Flt3 (Supplemental Fig. S2A) and frequent deletion of N-terminal Flt3 exons (Fig. 1). Furthermore, Northern blot analyses showed that CNSinvasive TM samples lacked expression of Flt3 exons 3-6 but expressed high levels of Flt3 exons 11-20, whereas expression of both Flt3 regions was undetectable in DM leukemias (Supplemental Fig. S2B,C). Using a ratiometric quantitative RT-PCR (qRT-PCR) assay, we confirmed that TM B-ALLs expressed very low levels of mRNA containing 5' Flt3 exons (Fig. 2B). However, they expressed significantly higher relative levels of Flt3 exons 11-24 compared with the $\mathrm{EBF}^{-/-}$progenitor cell line expressing full-length Flt3 (Gwin et al. 2010). Similar trFlt3 transcripts lacking exons 1-9 were associated with all three types of chromosome 5 abnormalities: trisomy, telomeric amplification, and der(2)(t $(2 ; 5)$. However, we did not detect FLT3-ITD or other known activating mutations (Frohling et al. 2007) in Flt3 transcripts from TM B-ALLs (data not shown).

TM B-ALLs isolated from mice with clinical signs of CNS leukemia expressed trFlt3 proteins with apparent molecular weights (MWs) much lower than that of full-
A

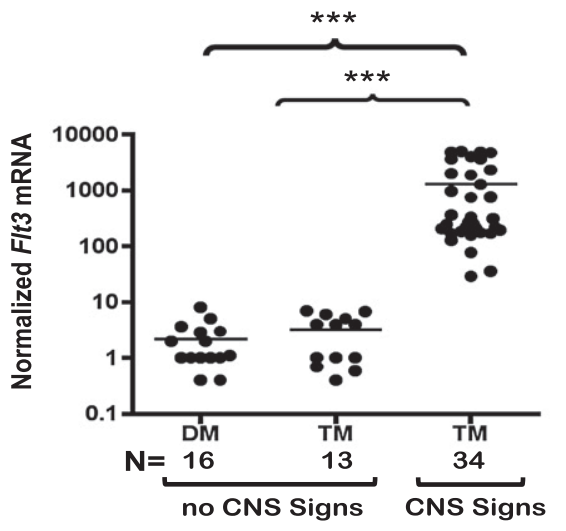

B

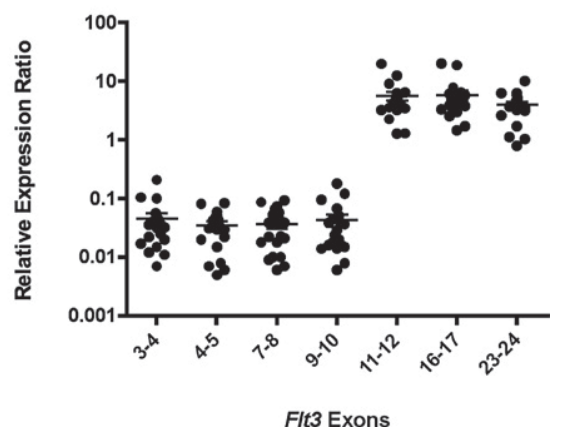

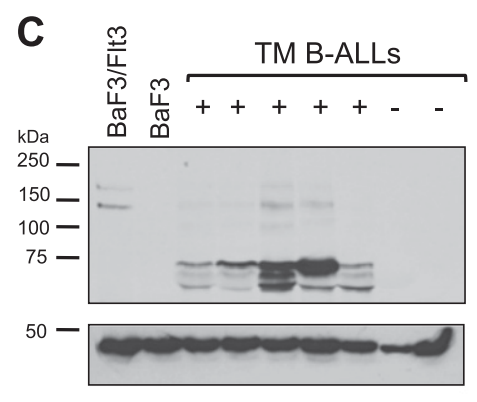

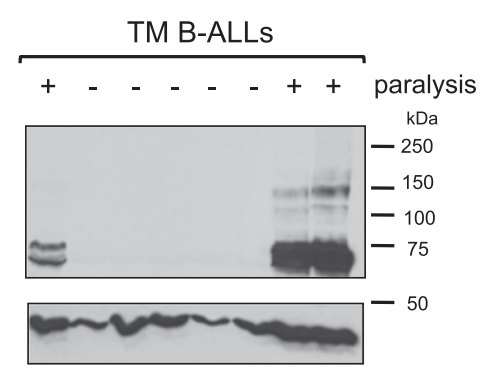

D

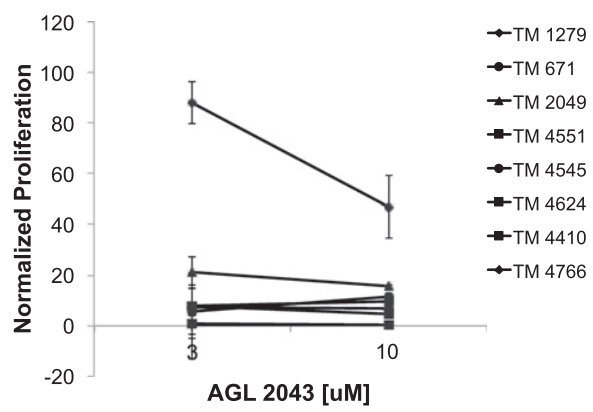

Figure 2. Expression and function of trFlt3. (A) mRNA was isolated from TM and DM leukemias that arose in mice with or without clinical signs of CNS leukemia and was subjected to qRT-PCR to measure Flt3 abundance (normalized to $\beta$-actin). Asterisks indicate statistically significant differences $(P<0.0001$, ANOVA Kruskal-Wallis test). (B) Ratiometric qRT-PCR analysis of Flt3 transcripts in TM B-ALLs. cDNA from TM B-ALLs or EBF1 $1^{-1-}$ cells was amplified using primer pairs spanning the indicated adjacent exons. Each measurement was made in triplicate, and threshold cycle values for each Flt3 amplicon were normalized to the $\beta$-actin threshold value for that sample to obtain relative expression ratios. Results are shown for 20 TM B-ALLs from mice with CNS signs. (C) Western blotting of Flt3 (top) and $\beta$-actin (bottom) protein in TM B-ALLs isolated from mice with $(+)$ or without $(-)$ paralysis as well as BaF3 and BaF3/Flt3 cells. Data shown are representative of 19 TM B-ALLs from mice with CNS signs and five TM B-ALLs from mice without CNS signs. $(D)$ The impact of AGL 2043 on proliferation of purified CD19+ cells from eight CNS-invasive TM B-ALLs (solid lines) was assessed using ${ }^{3} \mathrm{H}$-thymidine. Normalized proliferation (AGL 2043/DMSO) \pm standard deviation (error bars) was calculated from triplicate cultures. 
length Flt3 expressed in BaF3 progenitor cells (Fig. 2C). In contrast, TM B-ALLs isolated from mice lacking CNS leukemia did not express trFlt 3 protein, consistent with their low expression of Flt3 exons 11-24 (Fig. 2B). Using in silico tools, we identified an in-frame ATG with an adequate Kozak sequence in exon 10, corresponding to methionine residue M431 near the C-terminal end of the Ig4 domain. Two in-frame ATG codons in exon 12 (M504 and M521 in Ig5) could also potentially be used to translate chimeric ERV-Flt3 transcripts. The sizes of trFlt3 proteins roughly corresponded to those predicted for translation products initiating at M431, M504, or M521. Importantly, the ATP-competitive Flt3 inhibitor AGL 2043 significantly impaired FL-independent proliferation of seven out of eight primary leukemic TM B cells ex-vivo (Fig. 2D), with an IC $_{50}$ similar to that of the FLT3-ITDdependent MV4-11 AML cell line (Supplemental Fig. S2D). Collectively, these data suggest that CNS-invasive TM B-ALLs depend on a ligand-independent active form of Flt3 lacking mutations commonly found in human AML and B-ALL.

\section{ERV-Flt3 fusion transcripts and genes in TM B-ALLs}

The above findings suggested that the transcriptional start site for trFlt3 in TM B-ALLs was located between exons 10 and 12 . To test this possibility, we performed 5 ' rapid amplification of cDNA ends (RACE) on four TM B-ALL samples using a reverse primer anchored in Flt3 exon 12. Unexpectedly, sequencing of the cloned RACE products identified MuLV-related LTR sequences fused in-frame to Flt3 exon 10 (Fig. 3A). In all cases, Flt3 exons 1-9 were replaced by the Redundancy $(R)$ and Unique 5' (U5) regions of $E R V L T R$ s, with transcriptional start sites in $R$. Interestingly, there were two distinct $R / U 5$ sequences mismatched at eight of the first 112 nucleotides (nt) (data not shown), suggesting involvement of at least two different ERVs. In addition, each RACE product contained "ACTATAT" nucleotides of unknown origin just upstream of Flt3 exon 10 (see below).

We used RT-PCR to screen a cohort of 18 independent TM B-ALL samples for ERV-Flt3 fusion transcripts with a sense LTR primer coupled with an antisense primer anchored in Flt3 exon 12. All samples expressed ERV-Flt3 fusion transcripts (Fig. 3B; data not shown). We identified two major types of U5/Flt3 exon 10 junctions (Fig. 3C). Type 1 transcripts (11 out of 18 samples) contained an $E R V$ sequence $3^{\prime}$ of the LTR up to the proviral splice donor (SD). A single-nucleotide polymorphism (SNP) between the primer-binding site (PBS) and SD suggested the involvement of two different $E R V$ donors. In contrast, seven out of 18 samples had type 2 transcripts containing

\section{A ERV-LTR Sequence:}

GCGCCAGTCCTTCGATAGACTGAGTCGCCCGGGTACCCGTGTATCCAATA AAGCCTTTTGCTGTTGCATCCGAATCGTGGTCTCGCTGATCCTTGGGAGG GTCTCCTCAGAGTGATTGACTGCCCAGCCTGGGGGTCTTTCATTACTATAT

B
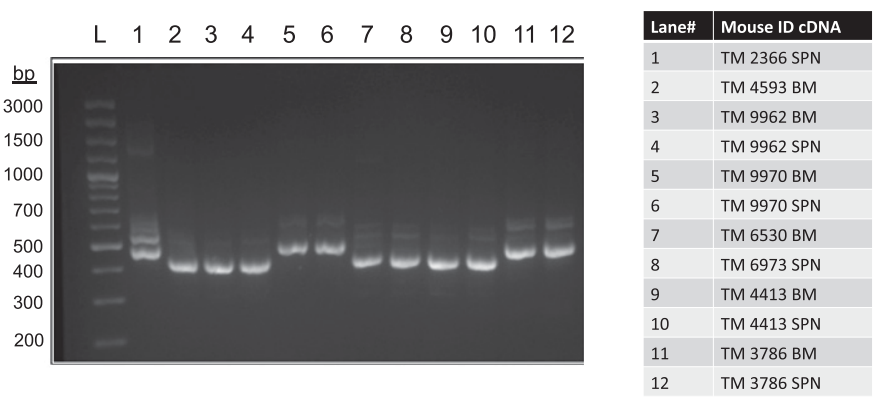

C

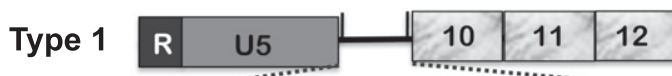

$(11 / 18)$

TGGGGGCTCGTCCGGGATTTGGAGACCCCCACCCAAGGACCACCGACCCACCGTGGGAG 5/11 TGGGGGCTCGTCCGGGATTTGGAGACCCCCËCCCAGGGACCACCGACCCACCGTGGGAG 6/11

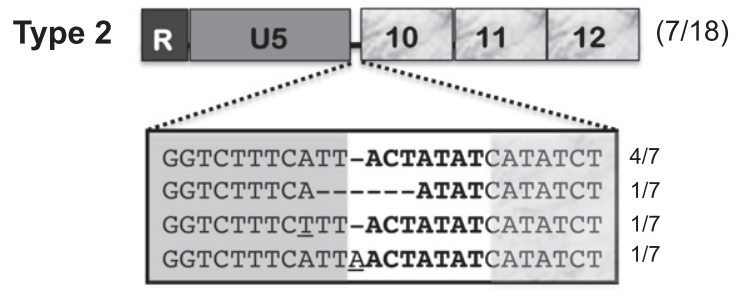

Figure 3. ERV-Flt3 fusion transcripts in TM B-ALLs. (A) One-hundred-forty-four nucleotides from cloned TM 6530 RACE product showed $99 \%$ identity $\left(P=9 \times 10^{-69}\right)$ with the $L T R / R$ (black) and LTR/U5 (gray) regions from MuLVrelated ERV sequences from accession numbers U63133, JO1997, JO1998, and M19118. The remaining $355 \mathrm{nt}$ aligned perfectly with Flt3 exons 10-12 in accession number NM010229, nucleotides 1433-1785 (not shown), except for the ACTATAT sequence (italics) just upstream of Flt3 exon 10. (B) RT-PCR analysis of ERV-Flt3 transcripts in TM B-ALL samples using LTR R/U5 forward (underlined in A) and Flt3 exon 12 reverse primers. (Lane $L$ ) One-kilobase DNA ladder. (Lanes 1-12) RNA from eight independent TM B-ALLs (both BM and spleen were analyzed for four mice). Results are representative of 18 TM B-ALLs analyzed. $(C)$ Schematic representation of type 1, type 2, and type 3 ERV-Flt3 fusion transcripts detected by sequencing cloned $5^{\prime}$ RACE and RT-PCR amplicons. Boxes display nucleotides at $U 5 /$ exon 10 junctions. For type 1 transcripts, proviral PBS and SD sequences are in bold. Nucleotides derived from LTR/U5 (dark gray) and Flt3 exons 10-12 (light gray) are indicated as well as the ACTATAT sequence of unknown origin (bold). SNPs are underlined. The frequency of samples expressing each type of transcript is indicated in brackets.

\begin{tabular}{|l|l|l|l|l|l|} 
Type 3 & $R$ & U5 & 10 & 11 & 12 \\
\hline
\end{tabular} 
Johnson et al.

ACTATAT or "ATAT" nucleotides of unknown origin at their U5/Flt3 junctions and also small additions or deletions in some cases. We detected another SNP at the 5' end of $U 5$, again suggesting two different $E R V$ donors. Some type 1 and type 2 samples also contained type 3 transcripts in which the $5^{\prime}$ part of $U 5$ was fused directly to Flt3 exon 10 (Fig. 3C; Supplemental Fig. S3). These findings suggest that trFlt3 transcripts arose from genomic rearrangements that deleted Flt3 exons 1-9 and juxtaposed ERV LTR-based transcriptional control elements with Flt3 exons 10-24.

To identify genomic rearrangements involving $E R V$ and Flt3 as well as the potential source of the ACTATAT insertions, we screened genomic DNA from TM B-ALL samples using a PCR strategy with $R / U 5$ sense and nested Flt3 antisense primers anchored in exon 10 and intron 10. ERV-Flt3 amplicons were not obtained from TM B-ALLs with type 1 transcripts, suggesting that the ERV was inserted too far upstream of Flt3 exon 10 to be detected by this PCR strategy. We did identify ERV-Flt3 fusion genes in TM B-ALLs that expressed type 2 transcripts (Fig. 4A).
ERV LTR sequences inserted into the same Flt3 intron 9 location in both samples, immediately upstream of an ACTATAT sequence. Thus, the $\sim 9-\mathrm{kb}$-long intron 9 was the likely origin of these nucleotides in type 2 transcripts.

The ACTATAT sequence in the ERV-Flt3 fusion gene from TM 4593 was included in a block of $112 \mathrm{nt}$ from intron 9, followed by a second block of intron 9 sequence from the region immediately adjacent to exon 10 (Fig. 4A, top). The intron 9 nucleotides immediately downstream from ACTATAT in the fusion gene included a potential SD sequence and likely functioned to allow splicing of the R/U5 LTR region into Flt3 exon 10. In contrast, ACTATAT was the only intron 9 sequence retained in the TM 6530 fusion gene (Fig. 4A, bottom), so this chimeric transcript was likely generated by readthrough transcription from U5 to Flt3 exon 10. Thus, the two samples exhibited distinct large interstitial deletions both upstream of and downstream from the ACTATAT insertion site in intron 9, indicating that they arose from different complex multistep genomic ERV-Flt3 rearrangements. Preservation of ACTATAT or ATAT sequence in different samples
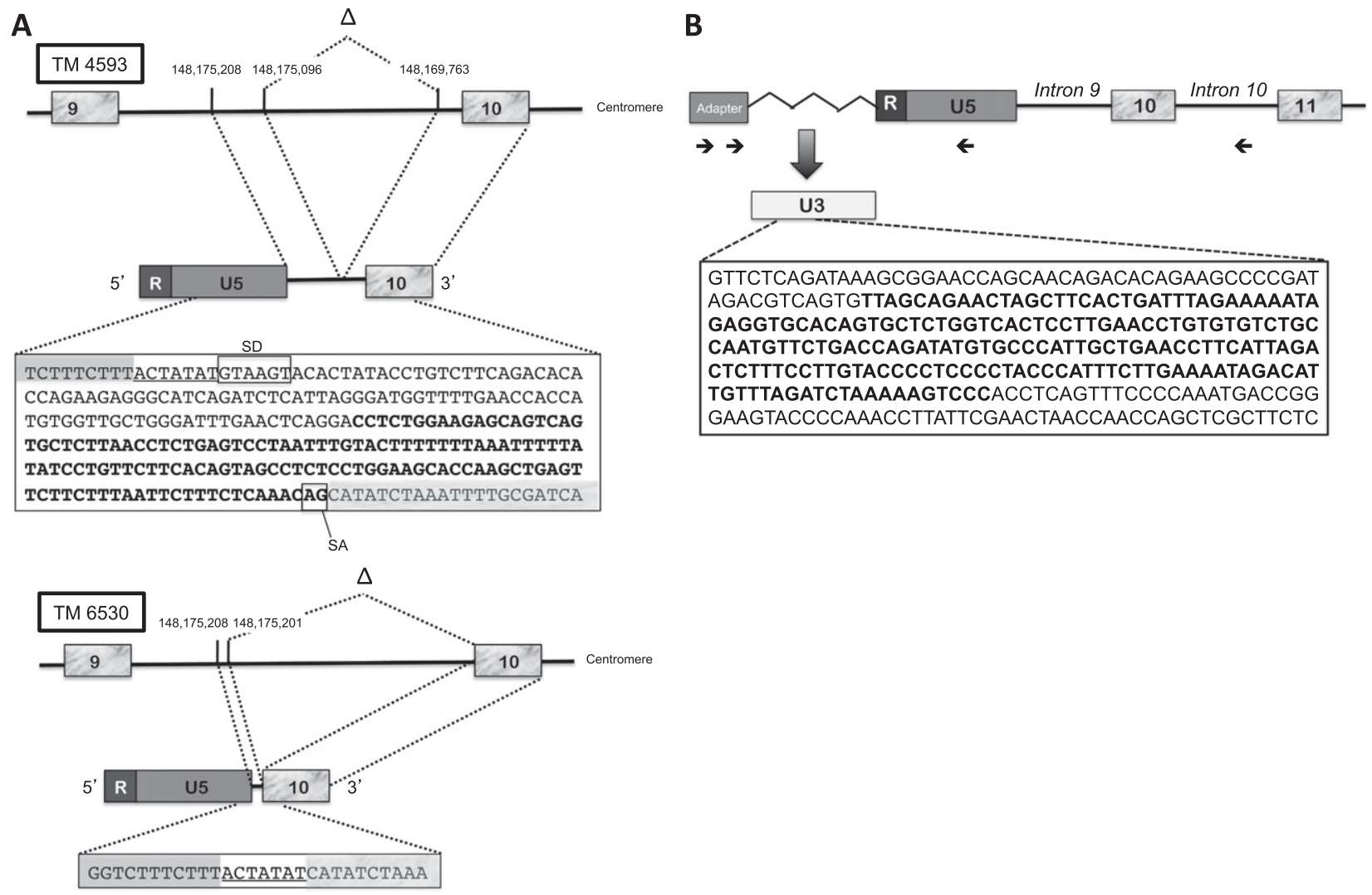

Figure 4. ERV-Flt3 gene fusions in TM B-ALL. (A) Schematics of ERV-Flt3 fusion genes in TM B-ALL 4593 (top) and 6530 (bottom) showing ERV-Flt3 junction sequences of amplicons cloned from genomic DNA. Numbers refer to chromosome $5(-)$ strand positions according to NCBI37/mm9. Sequences derived from the LTR/U5 region (dark gray) versus exon 10 (light gray) are highlighted. Intron 9 sequences in TM 4593 fusion genes are shown in regular font $(148,175,096-148,175,208)$ or bold font $(148,169,628-148,169,763)$. Putative SD and splice acceptor (SA) sequences are boxed. Intron 9 ACTATAT residues $(148,175,208-148,175,201)$ in both fusion genes are underlined. (B) LM-PCR strategy to identify the U3 sequence upstream of R/U5 in ERV-Flt3 fusion genes (primer locations are indicated by arrows). BLAST searches of cloned LM-PCR products (466 nt) yielded a perfect match with a modified polytropic U3 sequence (accession no. M17327), including a 190-nt insert (bold) found only in polytropic ERVs (Stoye and Coffin 1987). The same sequence was obtained in three independent TM B-ALLs. 
suggests that it has an important function, most likely because it is adjacent to a cryptic SD needed to generate chimeric ERV-Flt3 transcripts (Supplemental Fig. S3B).

We identified a $U 3$ element upstream of $R / U 5$ elements by performing ligation-mediated (LM) PCR with nested 3 ' primers anchored in Flt3 intron 10 and the R/U5 LTR sequence (Fig. 4B). In all three fusion genes, the $U 3$ element contained a 190-nt insert found only in polytropic ERVs (Stoye and Coffin 1987). About 40 partial or complete polytropic ERVs are found in most mouse strains, making them the most abundant class of MuLVrelated ERVs (Stocking and Kozak 2008). The ERV/U3 sequence that we identified in TM B-ALL fusion genes perfectly matched $13 E R V \mathrm{~s}$ in the mouse genome, including four on chromosome 5 (data not shown). Thus, there are many potential chromosomal sources for the ERVs found fused to Flt3 in TM B-cell leukemias.

Collectively, these data suggest that there was recurrent selection of B-cell progenitors harboring ERV-Flt3 fusion genes that could generate R/U5-Flt3 exon 10-24 chimeric transcripts by splicing events that use either a proviral (type 1) or cryptic (type 2) intron 9 SD. These splicing events allow expression of chimeric ERV-Flt3 transcripts (Supplemental Fig. S3) that promote aberrant survival and expansion of TM B-cell progenitors. Since there is a potential SD within the LTR/U5 region, type 3 transcripts likely arose by alternative splicing from type 1 or type 2 transcripts.

\section{Ligand-independent signaling by trFlt3}

The Flt3 exons missing from ERV-Flt3 fusion transcripts encode the first three immunoglobulin-like domains of Flt3 (Ig1-3), a region important for FL binding (Verstraete et al. 2011), as well as most of Ig4. Therefore, we hypothesized that loss of these regions may disrupt the autoinhibited conformation of the Flt3 kinase domain, allowing trFlt3 to signal constitutively. To test this notion, we transduced BaF3 cells with an MSCV-IRES-GFP (MIGR) retroviral vector expressing trFlt3 (exons 10-24), fulllength Flt3, or FLT3-ITD. The apparent MW of MIGR transduced trFlt3 was similar to trFlt3 expressed by TM B-ALLs (Fig. 5A). We used phospho-flow cytometry to analyze signaling properties of trFlt3 versus full-length murine Flt3 and human FLT3-ITD in IL3-dependent BaF3 hematopoietic progenitor cells. As expected (Choudhary et al. 2007), IL3, but not FL, induced STAT5 phosphorylation (pSTAT5) in BaF3/Flt3 cells, whereas both cytokines robustly increased pAKT, pS6, and pERK1/2, indicating robust activation of PI-3K and MAPK signaling (Fig. 5B). BaF3/FLT3-ITD and BaF3/trFlt3 cells exhibited increased basal (FL-independent) phosphorylation of these proteins that was diminished by acute treatment with AC220, a highly selective FLT3 inhibitor (Fig. 5C; Zarrinkar et al. 2009). In contrast, BaF3/MIGR cells did not exhibit detectable basal phosphorylation of STAT5, AKT, S6, or ERK1/2, suggesting that FLT3-ITD and trFlt3 specifically enhanced basal (FL-independent) activation of these signaling networks in BaF3 cells. Primary TM B-ALL blasts also expressed very high basal levels of AC220-sensitive
pSTAT5 (Fig. 5C, bottom), suggesting that trFlt3 promotes FL-independent STAT5 activation in TM B-ALL cells. Finally, expression of trFlt3 conferred IL3- and FLindependent growth to BaF3 cells, and this was substantially more robust than that induced by FL or FLT3ITD signaling (Fig. 5D). Importantly, AC220 abrogated FL-independent proliferation driven by both FLT3-ITD and trFlt3. Thus, trFlt3 and FLT3-ITD displayed similar ligand-independent STAT5 activation and conferred ligand-independent growth to BaF3 cells.

\section{TrFlt3 promotes development of CNS-invasive B-ALL}

We asked whether ectopic trFlt3 could promote development of CNS-disseminating B-ALLs by transducing fetal liver hematopoietic progenitors from DM mice with MIGR or MIGR/trFlt3 and transplanting them into $\mathrm{Rag}^{-/-}$hosts. Control MIGR-infected DM progenitor cells engrafted robustly and generated $\mathrm{CD}{ }^{+}$progeny 8 wk after secondary transplant (Fig. 6A,B). However, $\mathrm{CD} 19^{+}$cells were often $\mathrm{GFP}^{-}$, indicating that cells derived from MIGR-infected DM progenitors had no selective advantage over uninfected progenitors. Only one recipient had an enlarged spleen indicative of leukemia, but the leukemic cells did not invade the CNS.

In striking contrast, all recipients of MIGR/trFlt3transduced DM progenitors developed clinical signs of leukemia 8 wk after secondary transfer, and most $\mathrm{CD} 19^{+}$ progeny were $\mathrm{GFP}^{+}$(Fig. 6A,B), suggesting strong selection for trFlt3 expression. Furthermore, all recipients exhibited clinical signs of CNS leukemia and had leukemic B cells in the CNS (Fig. 6B,C). Finally, recipients of MIGR/trFlt3-transduced DM progenitors generally had higher numbers of splenic donor-derived B cells. Importantly, only MIGR/trFlt3-infected DM progenitors consistently generated full-blown B-ALL in these adoptive transfer experiments. Collectively, these findings demonstrate that trFlt 3 accelerates development of B-cell leukemia from p53/NHEJ-deficient hematopoietic progenitors and endows leukemic B cells with the capacity for CNS dissemination.

\section{Discussion}

In this study, we demonstrated that MuLV-related ERV sequences participate in aberrant end-joining events that promote leukemic transformation of B-cell precursors in p53/NHEJ-deficient mice. We identified a novel class of Flt3-activating mutations in CNS-invasive B-ALLs that were created by complex multistep chromosomal rearrangements with MuLV-related ERVs. N-terminal Flt3 exons encoding most of the ligand-binding domain were deleted and replaced with $E R V$ transcriptional control elements. Genomic instability conferred by p53/NHEJ deficiency likely facilitated the generation of these complex $E R V$-Flt3 fusion genes in TM B-ALLs. However, ERVFlt3 fusion genes were never detected in DM B-ALLs, revealing that Rag nuclease activity profoundly alters the mutational spectrum and thus clinical course of B-cell leukemogenesis caused by p53/NHEJ deficiency. Ectopic 
Johnson et al.
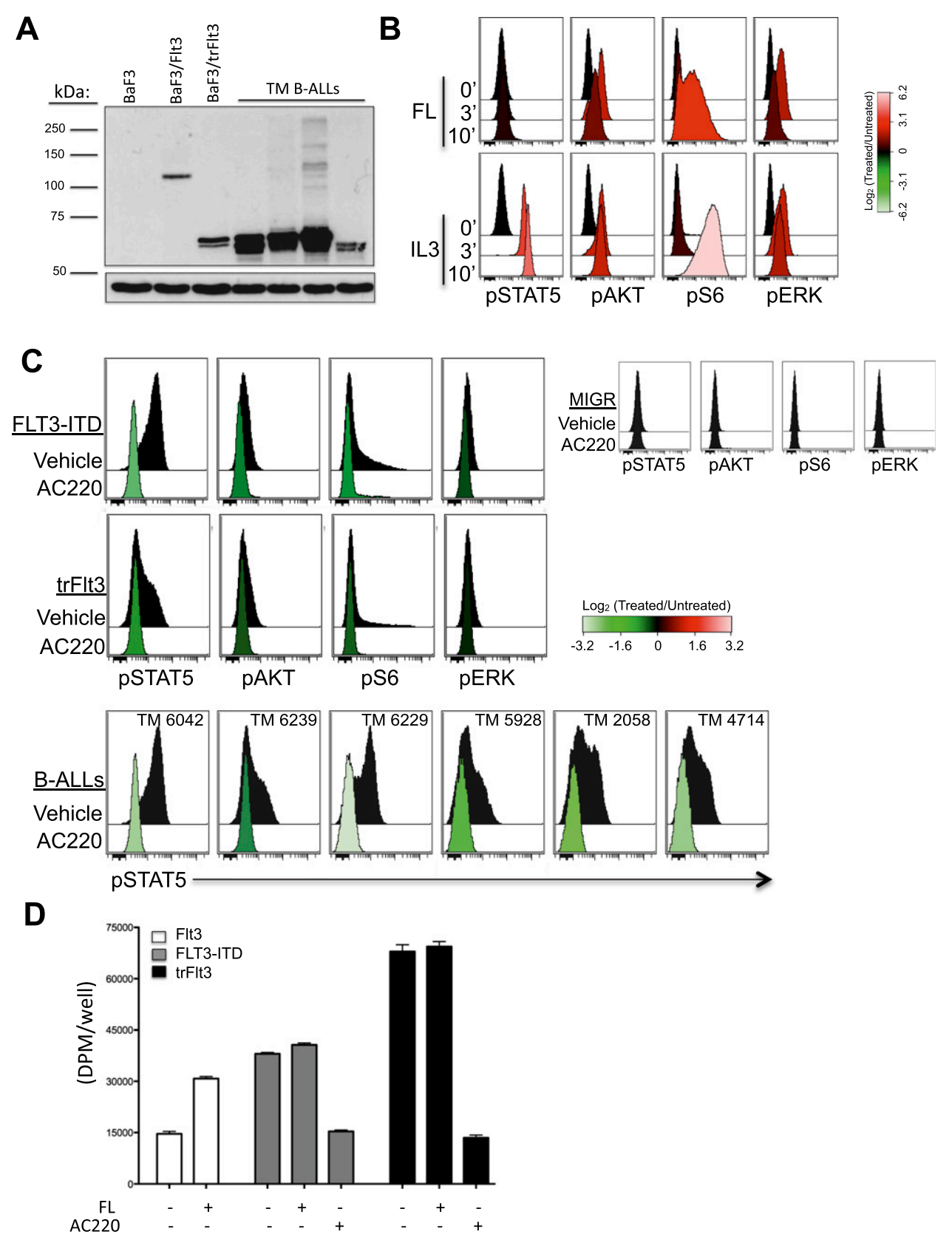

Figure 5. trFlt3 induces FL-independent signaling and proliferation of hematopoietic BaF3 cells. (A) Immunoblot detection of fulllength and trFlt3 expressed in BaF3 cells compared with CNS-invasive TM B-ALLs. (B) FL- versus IL3-induced signaling in BaF3 cells. IL3-starved BaF3/Flt3 cells were stimulated with FL or IL3 for 3 or 10 min prior to staining with phospho-specific antibodies. Histogram overlays show phospho-specific antibody staining after pregating on live single cells. Scales were based on $\log _{2}$ fold change in the MFI ratio of cytokine-treated/untreated cells. (C) Phospho-flow analysis of AC220-sensitive basal (without added cytokine) signaling in BaF3/MIGR, BaF3/trFlt3, BaF3/FLT-ITD, and leukemic TM B cells. Histogram overlays of TM B-ALL samples were pregated on live $\mathrm{CD} 1 \mathrm{~b}^{-} \mathrm{B} 220^{+}$singlets. Color scaling is based on the $\log _{2}$ MFI ratio of AC220-treated/untreated samples. $(D)$ trFlt3 confers IL3independent growth of BaF3 cells. BaF3 cell lines were cultured with $100 \mathrm{ng} / \mathrm{mL}$ FL or $1 \mathrm{nM} \mathrm{AC220}$ versus DMSO for $48 \mathrm{~h}$ prior to assessing proliferation. Data are displayed as the mean \pm standard deviation of triplicate measurements $\left(\mathrm{H}^{3}\right.$-thymidine disintegrations per minute $[\mathrm{DPM}]$ per well).

expression of trFlt3 promoted rapid generation of CNSdisseminating B-ALLs from DM hematopoietic progenitors, demonstrating that aberrant Flt3 activation underlies the unique ability of B-ALLs arising in TM mice to invade the CNS.
The propensity of exogenous MuLV to promote leukemogenesis by integrating near cellular proto-oncogenes is well established (Uren et al. 2005). Interestingly, mutation and recombination can sometimes promote generation of "reactivated" infectious ERVs in immune-deficient mice 
A

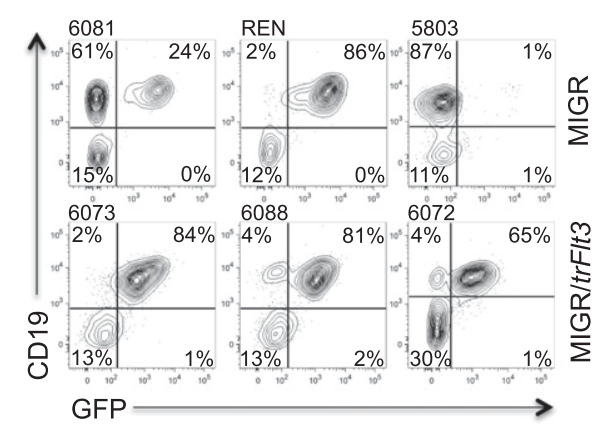

B

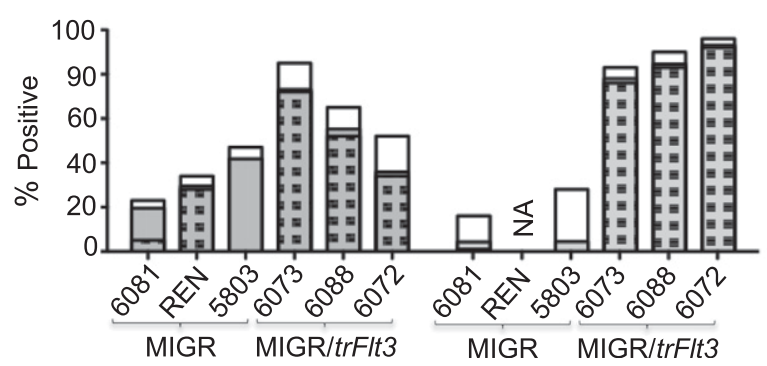

C

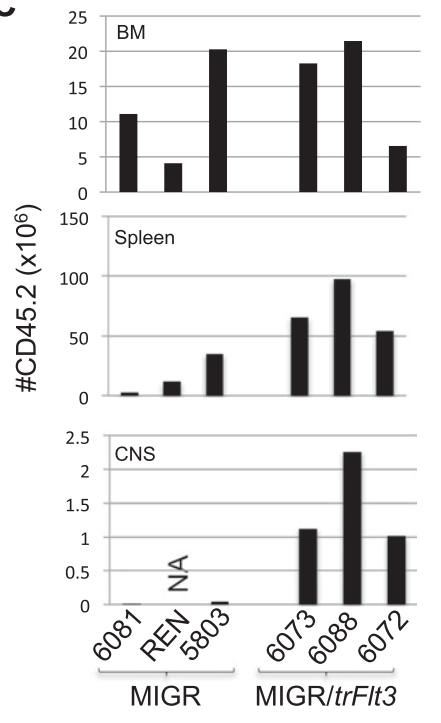

Figure 6. trFlt3 promotes CNS dissemination of B-ALL. BM, spleen, and CNS cells harvested from secondary Rag2 $2^{-/-}$recipients of MIGR or MIGR/trFlt3 transduced DM progenitors were analyzed by flow cytometry. (A) Contour plots (5\% probability) display CD19 versus GFP expression pregated on live single CD45.2+ donor-derived BM cells from three individual secondary recipients of DM progenitors transduced with MIGR (top) or MIGR/trFlt3 (bottom) retrovirus. (B) Frequency of total CD45.2 ${ }^{+}$donor cells (white), donor cells that were $\mathrm{CD} 19^{+} \mathrm{GFP}^{-}$(gray), and donor cells that were $\mathrm{CD}^{+} 9^{+} \mathrm{GFP}^{+}$(speckled) in each tissue. $(C)$ The number of donor-derived CD45.2 $2^{+}$cells in the BM (top), spleen (middle), and CNS (bottom). (NA) Not analyzed.

(Stoye et al. 1991; Young et al. 2012; Yu et al. 2012). However, type 2 ERV-Flt3 fusion genes lacked proviral PBS and SD sequences and were generated from complex, multistep rearrangements that were not consistent with simple integration of infectious $E R V$. Importantly, maintaining immune-deficient mice on acidified water alters intestinal microbial flora and suppresses $E R V$ reactivation by four to six orders of magnitude (Young et al. 2012). Since our DM and TM mice (and their progenitor strains) have been maintained on acidified water for $>10$ years, it is highly improbable that this ERV reactivation mechanism is operative in our colony. It is also unlikely that genetic background differences account for the preferential generation of ERV-Flt3 fusions in TM versus DM mice. The colony that generated the DM and TM mice used in this study has been interbreeding for $>10$ years, creating a recombinant inbred strain background segregating the p53 and Rag2 knockout alleles, effectively eliminating the possibility that genetic background differences account for the preferential generation of ERV-Flt3 fusions in TM mice.

Since NHEJ-deficient cells are more reliant on homology-based DSB repair mechanisms (McVey and Lee 2008), MuLV-related ERVs, present in 50-100 copies per genome, likely provide an abundant source of highly homologous sequences to facilitate DSB repair by nonallelic homologous recombination (Feschotte and Gilbert 2012), a mechanism that promotes chromosomal deletions, inversions, and translocations. We detected two SNPs in the non-LTR portion of type 1 fusions and four distinct LTR/U5 sequences, suggesting involvement of at least six distinct germline ERV elements. Thus, we suggest that NHEJ deficiency increases aberrant end-joining events involving repetitive MuLV-related ERVs. Furthermore, p53 deficiency prevents apoptosis triggered by DSB accumulation in NHEJ-deficient lymphocyte precursors (Guidos et al. 1996), allowing survival of NHEJ-deficient B-cell progenitors harboring ERV-Flt3 fusions or other aberrant end-joining events. Interestingly, ectopic Flt3 activation promotes apoptosis of CD19+ B-cell progenitors (Holmes et al. 2006), and p53 limits generation of leukemias induced by integration of exogenous MuLV near Flt3 (Uren et al. 2008). Therefore, we suggest that p53 deficiency enhances survival and selection of NHEJ-deficient B-cell progenitors ectopically expressing high levels of Flt3 under the control of ERV transcriptional control elements.

p53 deficiency also enhances survival and selection of B-cell progenitors harboring Rag-induced Igh/cMyc translocations in NHEJ-deficient mice (Difilippantonio et al. 2002; Zhu et al. 2002; Gladdy et al. 2003). We suggest that in DM mice, Igh/cMyc translocations are favored over ERV-Flt3 rearrangements because the former events rely on Rag-induced DSBs in Igh, which occur in all DM B-cell progenitors, whereas generation of ERV-Flt3 fusions would rely on random DSBs in both loci and are thus likely to occur less frequently. Since Rag deficiency prevents induction of DSBs in the Igh locus in TM B-cell progenitors, we suggest that rarer cells with ERV-Flt3 rearrangements undergo strong selection. 
Telomere capping and maintenance are also defective in NHEJ-deficient cells (d'Adda di Fagagna et al. 2001), so the telomeric location of murine Flt3 on the bottom strand of chromosome 5 likely enhances its susceptibility to this novel deletional mechanism of oncogenic activation. In some cases, the centromeric portion of Flt3 was amplified, suggesting that defective telomere maintenance in TM mice facilitated bridge-breakage-fusion cycles similar to those that generate complex and amplified Igh-cMyc translocations found in DM B-ALLs (Difilippantonio et al. 2002; Zhu et al. 2002; Gladdy et al. 2003). Similarly, defective telomere maintenance may explain the recurrent loss of telomeric chromosome 2 sequences in TM B-ALLs that were associated with $\mathrm{t}(2 ; 5)$ chromosomal translocations with breakpoints in Flt3. Thus, it seems likely that in addition to defective endjoining, telomere dysfunction also contributes to the generation of ERV-Flt3 fusions in TM B-cell progenitors.

We identified a novel mechanism for oncogenic Flt3 activation involving deletion of the extracellular FLbinding domain, recently localized to the $\operatorname{Ig} 3$ region (Verstraete et al. 2011). Flt3 lacks conserved residues involved in homotypic interactions between membraneproximal Ig4 and Ig5 domains that promote liganddependent activation of other class III receptor tyrosine kinases, and recent crystallographic data demonstrate the absence of such interactions upon FL binding to Flt3 (Verstraete et al. 2011). Flt3 has an atypical N-terminal Ig1 domain that adopts an unusual confirmation that may stabilize the autoinhibited receptor conformation in the absence of ligand. Deletion of exons encoding Flt3 Ig1-Ig4 domains likely destabilizes this autoinhibited conformation to promote FL-independent constitutive Flt3 activation. In support of this notion, we showed that, like FLT3ITD, trFlt3 induced FL-independent pSTAT5 and cytokine-independent proliferation of BaF3 cells.

Strikingly, TM B-ALLs expressing trFlt3 invaded the CNS and caused pathologies associated with CNS leukemia in humans, whereas DM as well as TM B-ALLs lacking trFlt3 did not disseminate to the CNS. Furthermore, retroviral transduction of trFlt3 promoted development of CNS-invasive B-cell leukemia from DM hematopoietic progenitors. These observations provide strong evidence that aberrant Flt3 signaling underlies the unique capacity of TM B-ALLs to disseminate to the CNS. Although FLT3 mutations are rare in B-ALL, expression of either wild-type or mutant FLT3 is frequent in $M L L$ rearranged infant B-ALL cases, which have high CNS relapse rates and poor overall survival (Isoyama et al. 2002; Nagayama et al. 2006; Pieters et al. 2007). Furthermore, high expression of wild-type FLT3 correlated with poor survival in infant B-ALL independent of $M L L$ rearrangement status (Kang et al. 2012). The blood-brain barrier prevents normal lymphocytes from entering the CNS, but inflammation associated with infection or autoimmunity allows lymphocytes to breach this barrier (Wilson et al. 2010). Thus, FLT3 activation may contribute to a proinflammatory environment that compromises the blood-brain barrier, enabling leukemic B cells to invade the CNS.
Surprisingly, TM mice did not develop AML or other nonlymphoid malignancies. The apparent lymphoid specificity of this $E R V$-induced oncogenesis most likely reflects the lymphoid specificity of MuLV-related transcriptional control elements, which would restrict expression of $E R V$ Flt3 fusion genes to lymphocyte precursors. This notion is consistent with the predominance of lymphoid leukemias induced by insertional mutagenesis after infection of newborn mice with MuLV (Uren et al. 2008). Thus, due to their lymphoid specificity and highly repetitive nature, MuLVrelated LTRs represent a major Rag-independent threat to genomic stability in p53/NHEJ-deficient B-cell precursors, and their aberrant repair can promote development of aggressive B-cell leukemia. Interestingly, ERV insertions have recently been observed in human malignancies (Tomlins et al. 2007; Lee et al. 2012), suggesting that human ERVs may also have oncogenic potential.

\section{Materials and methods}

\begin{abstract}
Mice
$p 53^{-/-}$Prkdc $c^{\text {scid/scid }}$ DM and $p 53^{-/-}$Rag2 $2^{-/-}$Prkdc $c^{\text {scid/scid }}$ TM mice were generated and genotyped as previously described (Gladdy et al. 2003). Briefly, DM mice were generated by breeding

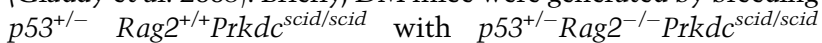
mice and selecting $p 53^{-/-}$Rag $2^{+/-}$Prkdc ${ }^{\text {scid/scid }}$ progeny. TM mice were generated by intercrossing $p 53^{+/-} \mathrm{Rag}^{-/-}$Prk $d c^{\text {scid/scid }}$ mice and selecting $p 53^{-/-} \mathrm{Rag}^{-/-}$Prk $d c^{\text {scid/scid }}$ progeny. The colony was maintained on acidified water, and mice were monitored daily for labored breathing and/or peripheral lymphadenopathy indicative of systemic leukemia as well as CNS signs, including a domed head, an ataxic gait, and hind limb paralysis and/or paresis. At the first sign of morbidity, mice were sacrificed, and single cells were isolated from lymphoid tissues or the CNS and used immediately or viably frozen in DMSO containing 90\% FCS and stored in liquid nitrogen. Samples were rapidly thawed and left to recover for $2 \mathrm{~h}$ at $37^{\circ} \mathrm{C}$ in StemSpan medium (Stem Cell Technologies) supplemented with $10 \%$ FBS before isolating nucleic acids or performing other experiments. All procedures were reviewed and approved by The Toronto Center for Phenogenomics Animal Care Committee.
\end{abstract}

\section{CGH and FISH}

Cy5-labeled DNA from leukemic cells and Cy3-labeled DNA from reference cells (livers from $p 53^{+/+}$Rag $^{-/-}$Prkdc ${ }^{\text {scid/scid }}$ sex mismatched littermates) were hybridized to mouse 6.5K RPCI23/-24 BAC arrays (http://www.roswellpark.org). Image captures and circular binary segmentation (CBS) analyses were performed using standard techniques. TM 5199 was also analyzed using mouse $385 \mathrm{~K}$ oligonucleotide whole-genome arrays (Roche NimbleGen). mFISH was performed using standard techniques with the following probes: RP23-355P12 (Spectrum Orange, Flt3 exons 3-24), RP23-102H13 (Spectrum Green, Flt3 exons 1-2), and RP23-68C11 plus RP23-349K12 BACs (Spectrum Aqua, chromosome 5 centromeric region).

\section{ERV-Flt3 fusion transcripts and genes}

5' RACE was performed on poly $(\mathrm{A})^{+}$RNA purified from four TM B-ALLs using the First Choice RLM-RACE kit (Ambion). Products were PCR-amplified using a 5' RACE adaptor and nested Flt3 antisense primers and were purified using the QIAquick 
PCR extraction kit (Qiagen). NCBI Open Reading Frame Finder and StarORF software were used to identify potential initiator methionine residues in ERV-Flt3 sequences. To identify fusion genes, genomic DNA was amplified using $E R V$ sense and nested Flt3 intron 10 (outer) followed by exon 10 (inner) antisense primers. For LM-PCR, PvuII-digested genomic DNA was ligated to GenomeWalker universal kit adaptors and amplified using outer primers specific for the adaptor and Flt3 intron 10, followed by nested inner primers for the adaptor and ERV/U5. All PCRs were performed with the Expand Long PCR template kit (Roche Diagnostics), and products were purified using the QIAquick PCR extraction kit and cloned into pDrive Vector using the Qiagen PCR Cloning Plus kit (Qiagen). Multiple clones from each leukemia sample were sequenced in both directions. Primer sequences are in Supplemental Table 1.

\section{Retroviral constructs and transduction}

MIGR/Flt3 (Holmes et al. 2006) served as a template to PCRamplify trFlt3 (exons 10-24). The exon 10 forward primer included a BamH1 site, a Kozak "ACC" sequence, and nucleotides complementary to the M431 ATG methionine codon in Flt3 exon 10 followed by 17 downstream nucleotides (Supplemental Table 2). The exon 24 reverse primer included a BamH1 recognition site as well as a stop codon. MIGR plasmids with correctly oriented Flt3 exon 10-24 inserts were identified by sequencing. Retroviruses were produced by transiently transfecting MIGR plasmids into ecotropic Platinum-E retroviral packaging cells (Cell Biolabs, Inc.) using standard techniques. Retrovirus-containing supernatants were harvested $48 \mathrm{~h}$ post-infection, filtered $(0.45 \mu \mathrm{m})$, titered on $\mathrm{NIH}-3 \mathrm{~T} 3$ cells, and stored at $-80^{\circ} \mathrm{C}$ prior to infecting BaF3 cells by two rounds of spinoculation (1000 $\mathrm{g}$ for $1.5 \mathrm{~h}, 2$-h recovery in between). Cells were cultured 3-4 d after transduction, and $\mathrm{GFP}^{+}$cells were isolated by cell sorting to establish stably transduced $\mathrm{BaF} 3$ cell lines. Sorted embryonic day 14.5 (E14.5) Ter119- $\mathrm{B}^{-220^{-}} \mathrm{Gr}-1^{-} \mathrm{CD}^{-} \mathrm{CD}^{-}$fetal liver hematopoietic progenitor cells from DM embryos were prestimulated overnight with growth factors $(100 \mathrm{ng} / \mathrm{mL}$ SCF and FL, 50 $\mathrm{ng} / \mathrm{mL}$ TPO, $5 \mathrm{ng} / \mathrm{mL}$ IL6 and IL11). Cells were transduced by two rounds of spinoculation in retroviral supernatant supplemented with growth factors and Transdux (System Bioscience) as per the manufacturer's recommendations and then resuspended in Hanks' balanced salt solution (Life Technologies) and injected intrafemorally $(400,000$ cells per mouse) into sublethally irradiated (650 cGy) Rag2 ${ }^{-/-}$mice. Five weeks later, bone marrow (BM) from hosts confirmed to contain $\mathrm{GFP}^{+}$donor-derived

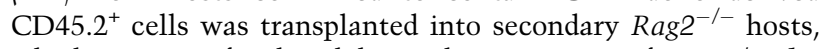
which were sacrificed 8 wk later when recipients of MIGR/trFlt3 transduced cells showed clinical signs of CNS leukemia.

\section{Phospho-flow cytometry}

For phospho-flow cytometry, BaF3 cell lines were precultured for $6 \mathrm{~h}$ at $37^{\circ} \mathrm{C}$ without IL3 in serum-free and phenol red-free RPMI1640 before stimulating with $10 \mathrm{ng} / \mathrm{mL}$ FL or IL3 for 3 or $10 \mathrm{~min}$. Viably frozen TM B-ALL samples were thawed and cultured for $1 \mathrm{~h}$ in StemSpan with 10\% FBS prior to starving for $2 \mathrm{~h}$ in serumfree and phenol red-free RPMI-1640. Where indicated, $5 \mathrm{nM}$ AC220 (Selleck Chemicals) or 0.002\% DMSO vehicle was added during the 2-h starvation period. Fixable Blue viability dye (Invitrogen) was added during the last $30 \mathrm{~min}$. Cells were then fixed (BD Cytofix), permeabilized (BD Phos-flow Buffer III), and stained with predetermined optimal concentrations of antibodies (BD Biosciences) specific for the following phosphoepitopes: pSTAT5 (pY694, clone 47), pAKT (pT308, clone J1223.371), pERK1/2 (pT202/Y204, clone 20a), and pS6 (pS235/
S236, clone N7-548). TM leukemic samples were also stained with CD11b (M1/70) or B220 (RA3-6B2). Fluorescence was quantified using a BD Fortessa flow cytometer, and FCS 3.0 data files were uploaded to FlowJo for pregating to exclude dead cells, doublets, and debris. Pregated data files were uploaded into Cytobank (http://www.cytobank.org), a cloud-based flow cytometry analytic platform, for statistical analysis and visualization.

\section{Acknowledgments}

We thank Kay Medina for $\mathrm{EBF}^{-/-}$cells, Stephen Nutt for MIGR/Flt3, and John Dick and Mark Minden for MIGR/FLT3ITD and MV4-11 cells, respectively. This work was supported by operating grants to C.J.G. and J.S.D. from the Ontario Institute for Cancer Research Cancer Stem Cells Program (with funds provided by the Government of Ontario), the Leukemia and Lymphoma Society USA, and Genome Canada through the Ontario Genomics Institute. R.M.J. received a Canadian Institutes of Health Research Canadian Graduate Scholarship and a Hospital for Sick Children Restracomp Studentship. E.P. received a graduate scholarship from the Ontario Institute for Cancer Research Cancer Stem Cells Program. Sequence and cytogenetic analyses were performed at The Centre for Applied Genomics, Hospital for Sick Children, with support from Genome Canada/Ontario Genomics Institute and the Canada Foundation for Innovation. Flow cytometry was performed in The SickKids-University Health Network Flow Cytometry Facility, supported by funding from the Ontario Institute for Cancer Research, McEwen Centre for Regenerative Medicine, Canada Foundation for Innovation, and SickKids Foundation.

\section{References}

Choudhary C, Brandts C, Schwable J, Tickenbrock L, Sargin B, Ueker A, Bohmer FD, Berdel WE, Muller-Tidow C, Serve H. 2007. Activation mechanisms of STAT5 by oncogenic Flt3ITD. Blood 110: 370-374.

d'Adda di Fagagna F, Hande MP, Tong WM, Roth D, Lansdorp PM, Wang ZQ, Jackson SP. 2001. Effects of DNA nonhomologous end-joining factors on telomere length and chromosomal stability in mammalian cells. Curr Biol 11: 11921196.

d'Adda di Fagagna F, Teo SH, Jackson SP. 2004. Functional links between telomeres and proteins of the DNA-damage response. Genes Dev 18: 1781-1799.

Difilippantonio MJ, Petersen S, Chen HT, Johnson R, Jasin M, Kanaar R, Ried T, Nussenzweig A. 2002. Evidence for replicative repair of DNA double-strand breaks leading to oncogenic translocation and gene amplification. I Exp Med 196: 469-480.

Feschotte C, Gilbert C. 2012. Endogenous viruses: insights into viral evolution and impact on host biology. Nat Rev Genet 13: $283-296$.

Frohling S, Scholl C, Levine RL, Loriaux M, Boggon TJ, Bernard OA, Berger R, Dohner H, Dohner K, Ebert BL, et al. 2007. Identification of driver and passenger mutations of FLT3 by high-throughput DNA sequence analysis and functional assessment of candidate alleles. Cancer Cell 12: 501-513.

Gladdy RA, Taylor MD, Williams CJ, Grandal I, Karaskova J, Squire JA, Rutka JT, Guidos CJ, Danska JS. 2003. The RAG$1 / 2$ endonuclease causes genomic instability and controls CNS complications of lymphoblastic leukemia in p53/Prkdcdeficient mice. Cancer Cell 3: 37-50.

Guidos CJ, Williams CJ, Grandal I, Knowles G, Huang MT, Danska JS. 1996. V(D)J recombination activates a p53-de- 
pendent DNA damage checkpoint in scid lymphocyte precursors. Genes Dev 10: 2038-2054.

Gwin K, Frank E, Bossou A, Medina KL. 2010. Hoxa9 regulates Flt3 in lymphohematopoietic progenitors. I Immunol 185: 6572-6583.

Holmes ML, Carotta S, Corcoran LM, Nutt SL. 2006. Repression of Flt3 by Pax5 is crucial for B-cell lineage commitment. Genes Dev 20: 933-938.

Inaba H, Greaves M, Mullighan CG. 2013. Acute lymphoblastic leukaemia. Lancet 381: 1943-1955.

Isoyama $\mathrm{K}$, Eguchi $\mathrm{M}$, Hibi $\mathrm{S}$, Kinukawa $\mathrm{N}$, Ohkawa $\mathrm{H}$, Kawasaki H, Kosaka Y, Oda T, Oda M, Okamura T, et al. 2002. Risk-directed treatment of infant acute lymphoblastic leukaemia based on early assessment of MLL gene status: results of the Japan Infant Leukaemia Study (MLL96). Br J Haematol 118: 999-1010.

Kang H, Wilson CS, Harvey RC, Chen IM, Murphy MH, Atlas SR, Bedrick EJ, Devidas M, Carroll AJ, Robinson BW, et al. 2012. Gene expression profiles predictive of outcome and age in infant acute lymphoblastic leukemia: a Children's Oncology Group study. Blood 119: 1872-1881.

Lee E, Iskow R, Yang L, Gokcumen O, Haseley P, Luquette LJ 3rd, Lohr JG, Harris CC, Ding L, Wilson RK, et al. 2012. Landscape of somatic retrotransposition in human cancers. Science 337: 967-971.

McVey M, Lee SE. 2008. MMEJ repair of double-strand breaks (director's cut): deleted sequences and alternative endings. Trends Genet 24: 529-538.

Mullighan CG, Miller CB, Radtke I, Phillips LA, Dalton J, Ma J, White D, Hughes TP, Le Beau MM, Pui CH, et al. 2008. BCRABL1 lymphoblastic leukaemia is characterized by the deletion of Ikaros. Nature 453: 110-114.

Nagayama J, Tomizawa D, Koh K, Nagatoshi Y, Hotta N, Kishimoto T, Takahashi Y, Kuno T, Sugita K, Sato T, et al. 2006. Infants with acute lymphoblastic leukemia and a germline MLL gene are highly curable with use of chemotherapy alone: results from the Japan Infant Leukemia Study Group. Blood 107: 4663-4665.

Papaemmanuil E, Rapado I, Li Y, Potter NE, Wedge DC, Tubio J, Alexandrov LB, Van Loo P, Cooke SL, Marshall J, et al. 2014 RAG-mediated recombination is the predominant driver of oncogenic rearrangement in ETV6-RUNX1 acute lymphoblastic leukemia. Nat Genet 46: 116-125.

Pieters R, Schrappe M, De Lorenzo P, Hann I, De Rossi G, Felice M, Hovi L, LeBlanc T, Szczepanski T, Ferster A, et al. 2007. A treatment protocol for infants younger than 1 year with acute lymphoblastic leukaemia (interfant-99): an observational study and a multicentre randomised trial. Lancet 370: 240-250.

Pui CH. 2006. Central nervous system disease in acute lymphoblastic leukemia: prophylaxis and treatment. Hematology Am Soc Hematol Educ Program 2006: 142-146.

Stirewalt DL, Radich JP. 2003. The role of FLT3 in haematopoietic malignancies. Nat Rev Cancer 3: 650-665.

Stocking C, Kozak CA. 2008. Murine endogenous retroviruses. Cell Mol Life Sci 65: 3383-3398.

Stoye JP. 2012. Studies of endogenous retroviruses reveal a continuing evolutionary saga. Nat Rev Microbiol 10: 395-406.

Stoye JP, Coffin JM. 1987. The four classes of endogenous murine leukemia virus: structural relationships and potential for recombination. J Virol 61: 2659-2669.

Stoye JP, Moroni C, Coffin JM. 1991. Virological events leading to spontaneous AKR thymomas. J Virol 65: 1273-1285.

Thompson CB. 1995. New insights into V(D)J recombination and its role in the evolution of the immune system. Immunity 3: 531-539.
Tomlins SA, Laxman B, Dhanasekaran SM, Helgeson BE, Cao X, Morris DS, Menon A, Jing X, Cao Q, Han B, et al. 2007. Distinct classes of chromosomal rearrangements create oncogenic ETS gene fusions in prostate cancer. Nature 448: 595-599.

Uren AG, Kool J, Berns A, van Lohuizen M. 2005. Retroviral insertional mutagenesis: past, present and future. Oncogene 24: 7656-7672.

Uren AG, Kool J, Matentzoglu K, de Ridder J, Mattison J, van Uitert M, Lagcher W, Sie D, Tanger E, Cox T, et al. 2008. Large-scale mutagenesis in p19(ARF)- and p53-deficient mice identifies cancer genes and their collaborative networks. Cell 133: 727-741.

Verstraete K, Vandriessche G, Januar M, Elegheert J, Shkumatov AV, Desfosses A, Van Craenenbroeck K, Svergun DI, Gutsche I, Vergauwen B, et al. 2011. Structural insights into the extracellular assembly of the hematopoietic Flt3 signaling complex. Blood 118: 60-68.

Wilson EH, Weninger W, Hunter CA. 2010. Trafficking of immune cells in the central nervous system. I Clin Invest 120: $1368-1379$.

Young GR, Eksmond U, Salcedo R, Alexopoulou L, Stoye JP, Kassiotis G. 2012. Resurrection of endogenous retroviruses in antibody-deficient mice. Nature 491: 774-778.

Yu P, Lubben W, Slomka H, Gebler J, Konert M, Cai C, Neubrandt L, Prazeres da Costa O, Paul S, Dehnert S, et al. 2012. Nucleic acid-sensing Toll-like receptors are essential for the control of endogenous retrovirus viremia and ERVinduced tumors. Immunity 37: 867-879.

Zarrinkar PP, Gunawardane RN, Cramer MD, Gardner MF, Brigham D, Belli B, Karaman MW, Pratz KW, Pallares G, Chao Q, et al. 2009. AC220 is a uniquely potent and selective inhibitor of FLT3 for the treatment of acute myeloid leukemia (AML). Blood 114: 2984-2992.

Zhu C, Mills KD, Ferguson DO, Lee C, Manis J, Fleming J, Gao Y, Morton CC, Alt FW. 2002. Unrepaired DNA breaks in p53-deficient cells lead to oncogenic gene amplification subsequent to translocations. Cell 109: 811-821. 


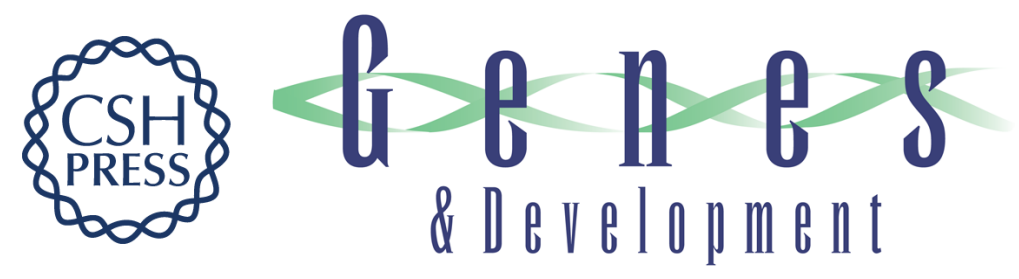

\section{MuLV-related endogenous retroviral elements and Flt3 participate in aberrant end-joining events that promote B-cell leukemogenesis}

Radia M. Johnson, Eniko Papp, Ildiko Grandal, et al.

Genes Dev. 2014, 28:

Access the most recent version at doi:10.1101/gad.240820.114 Supplemental
Material http://genesdev.cshlp.org/content/suppl/2014/05/28/28.11.1179.DC1

References This article cites 35 articles, 14 of which can be accessed free at: http://genesdev.cshlp.org/content/28/11/1179.full.html\#ref-list-1

Creative This article is distributed exclusively by Cold Spring Harbor Laboratory Press for the first Commons License

Email Alerting Service six months after the full-issue publication date (see http://genesdev.cshlp.org/site/misc/terms.xhtml). After six months, it is available under a Creative Commons License (Attribution-NonCommercial 4.0 International), as described at http://creativecommons.org/licenses/by-nc/4.0/.

Receive free email alerts when new articles cite this article - sign up in the box at the top right corner of the article or click here.

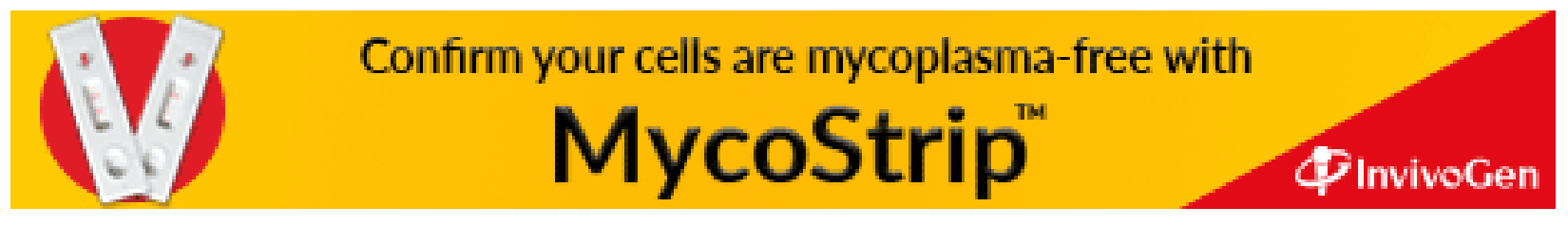

